\section{Afasia como debut de estado hiperosmolar hiperglicémico. Caso clínico}

\author{
SERGIO LEGUA KOC ${ }^{1}$, ALEJANDRO MARTÍNEZ GODOY ${ }^{1}$, \\ MANUEL ALVARADO PASTENES ${ }^{1,2}$, DAVID SÁEZ MÉNDEZ ${ }^{1,2}$
}

\section{Aphasia as the presenting symptom of a hyperglycemic hyperosmolar state. Case report}

Neurological manifestations such as seizures, disorders of consciousness and abnormal movements such as hemichorea and hemiballismus can be the presenting symptoms of hyperglycemic hyperosmolar states. Exceptionally, focal signs as hemiparesis or aphasia are described. We report a 66-year-old man, presenting with nonfluent aphasia and right subtle hemiparesis. The computed tomography, computed tomography angiography and brain magnetic resonance did not show acute ischemic lesions or obstruction of arterial vessels. The initial laboratory evaluation disclosed a blood glucose of $936 \mathrm{mg} / \mathrm{dL}$, a plasma osmolality of 331 $\mathrm{mOsm} / \mathrm{Kg}$, and positive plasma ketones. After the treatment of hyperglycemia and hyperosmolality, focal symptoms subsided.

(Rev Med Chile 2020; 148: 553-556)

Key words: Aphasia; Hyperglycemic Hyperosmolar Nonketotic Coma; Stroke.
'Departamento de Neurología

Sur, Facultad de Medicina,

Universidad de Chile. Santiago,

Chile.

${ }^{2}$ Servicio de Neurología. Complejo Asistencial Barros Luco Trudeau.

Servicio de Salud Metropolitano

Sur. Santiago, Chile

Trabajo no recibió financiamiento. Los autores declaran no tener conflictos de interés.

Recibido el 10 de octubre de 2019, aceptado el 22 de abril de 2020.

Correspondencia a:

Dr. Sergio Legua

Hospital Barros Luco Trudeau.

Gran Avenida José Miguel Carrera 3204, San Miguel. Santiago,

Chile.

sergiolegua@gmail.com
$\mathrm{E}$ l estado hiperosmolar hiperglicémico (EHH) es una complicación grave, secundaria en la mayoría de los casos a diabetes mellitus tipo 2, que se da principalmente en ancianos. Tiene menor frecuencia pero mayor mortalidad que la cetoacidosis diabética (10-20\%) y de allí la importancia de su sospecha y tratamiento precoz. Los criterios para EHH incluyen una hiperglicemia mayor a $600 \mathrm{mg} / \mathrm{dl}$, osmolaridad mayor a $320 \mathrm{mOsm} / \mathrm{kg}$, acidosis metabólica con $\mathrm{pH}$ mayor a 7,3 e hipercetonemia ${ }^{1}$. Usualmente presenta síntomas neurológicos, entre los cuales se encuentran principalmente el compromiso de conciencia, incluyendo desde la inatención hasta el coma; convulsiones y movimientos anormales, como hemibalismo y hemicorea ${ }^{2}$. La focalidad neurológica deficitaria es excepcional, siendo un diagnóstico diferencial de patología cerebrovascular, lo que se denomina en la literatura actual como simulador de infarto cerebral o "stroke mimic" en literatura anglosajona y que incluye síndromes convulsivos, migraña con aura, cuadros conversivos, entre otros ${ }^{3}$. A continuación presentamos un caso de un hombre con síntomas focales concomitantemente con un EHH.

\section{Caso clínico}

Se trata de un paciente masculino, de 66 años, dominancia manual diestra y autovalente, con antecedente de hipertensión arterial en tratamiento con enalapril $10 \mathrm{mg} /$ día. Acudió al servicio de urgencia del hospital Barros Luco Trudeau por presentar en forma ictal, mientras caminaba junto a su esposa, dificultad para emitir lenguaje y para comprender órdenes (lograba comprender órdenes de uno y ocasionalmente dos comandos), asociado a debilidad leve para movilizar el hemicuerpo derecho, pero que no impedía la marcha y que limitaba mínimamente la manipulación de objetos. Al llegar al servicio de urgencia, se constató presión arterial 
181/108 mmHg, frecuencia cardiaca 97 latidos por minuto, frecuencia respiratoria 19 ciclos por minuto y saturación de oxígeno $94 \%$ sin apoyo suplementario. Al examen físico general destacaba piel y mucosas secas y llene capilar conservado. Por alteración de lenguaje y debilidad de hemicuerpo derecho, se sospechó accidente cerebrovascular isquémico y por tiempo de evolución $(2 \mathrm{~h})$, se activó el protocolo de revascularización, que incluye la evaluación clínica inmediata por equipo de neurología, realización de neuroimágenes y toma de exámenes de laboratorio, donde se incluye en primer lugar la glicemia capilar, cuya medición fue "HI", lo cual traduce un valor sobre $600 \mathrm{mg} / \mathrm{dl}$. $\mathrm{Al}$ examen neurológico destacaba fluencia disminuida, nominación de 3 de 7 palabras, alteración grave de repetición y comprensión de órdenes escasa (dos comandos), ausencia de parafasias y mínima paresia a derecha, con hipotonía y reflejo cutáneo plantar indiferente ipsilateral. Se realizó tomografía computarizada (TAC) y angiografía por tomografía computarizada (angioTAC) de cerebro que no evidenciaron lesiones agudas isquémicas ni obstrucción de vasos arteriales. Se realizó resonancia magnética (RM) de cerebro con secuencias de Difussion-Weighted Imaging (DWI) y Apparent Diffusion Coefficient (ADC), que no evidenció lesiones agudas isquémicas, hemorragias ni lesiones secuelares (Figura 1). Se recibieron paralelamente los resultados de los exámenes plasmáticos por protocolo, que evidenciaron glicemia de 936 mg/dL, osmolaridad plasmática de $331 \mathrm{mOsm} /$ $\mathrm{kg}$, creatinina de $3 \mathrm{mg} / \mathrm{dL}$, natremia de $130 \mathrm{mEq} / \mathrm{l}$ (corregido por glicemia $143 \mathrm{mEq} / \mathrm{l}$ ), kalemia 4,2 $\mathrm{mEq} / \mathrm{l}$, cetonas plasmáticas positivas, $\mathrm{pH} \mathrm{7,37} \mathrm{y}$ bicarbonato de 19,3 mmol/l. Se desestima el tratamiento trombolítico y se inicia inmediatamente hidratación con solución de cloruro de sodio al 0,9\%, 1.000 mililitros ( $\mathrm{ml}$ ) en bolo y luego bomba de infusión continua a $100 \mathrm{a} \mathrm{ml} / \mathrm{h}$ e insulina cristalina en infusión continua a 7 unidades/h. Con el inicio del tratamiento se evidenció remi-
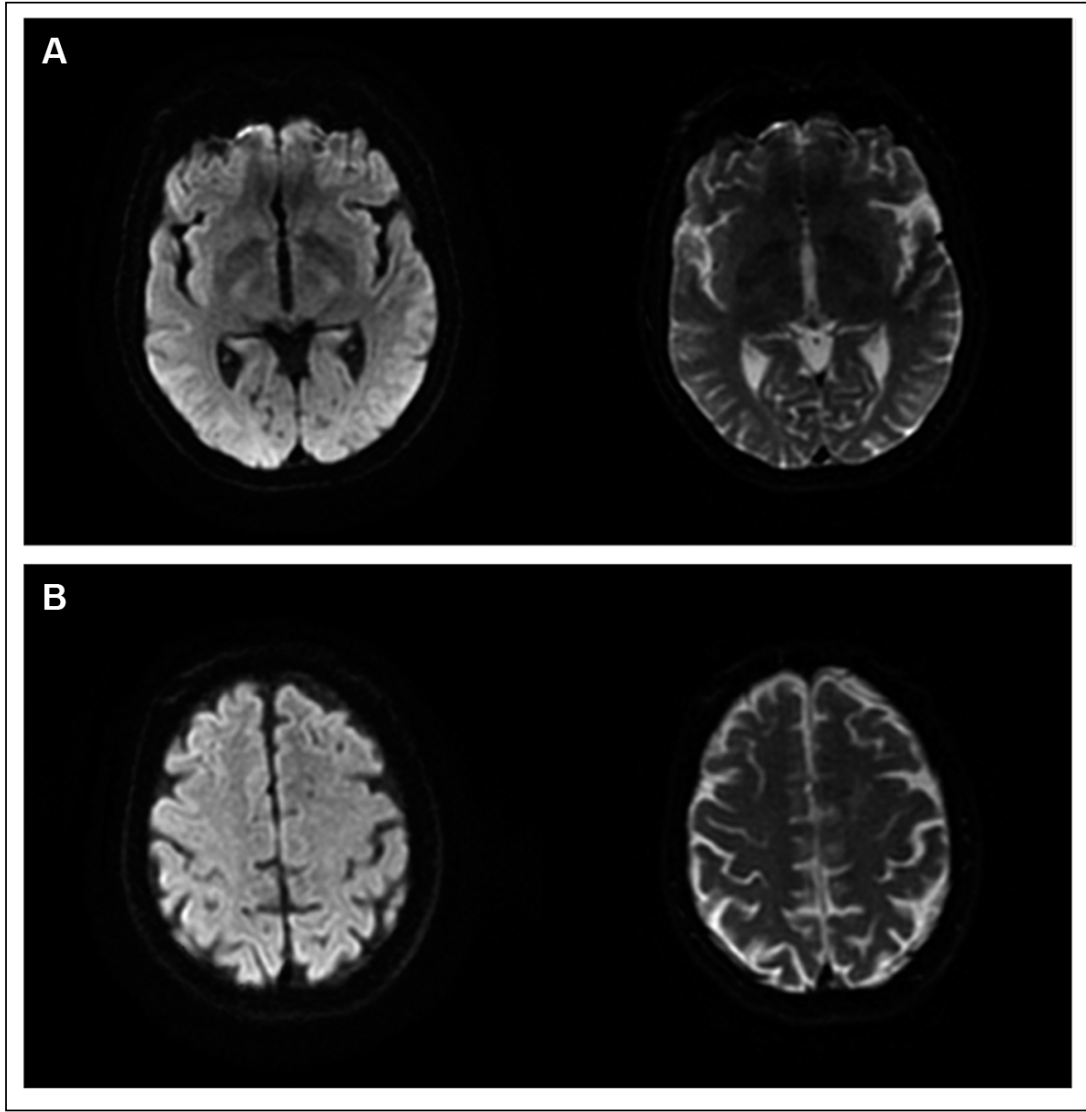

Figura 1. Resonancia magnética de cerebro. Secuencias de Difussion-Weighted Imaging (DWI)y Apparent Diffusion Coefficient (ADC). Corte axial en región temporal (A) y frontal (B) que no evidencia alteraciones. 


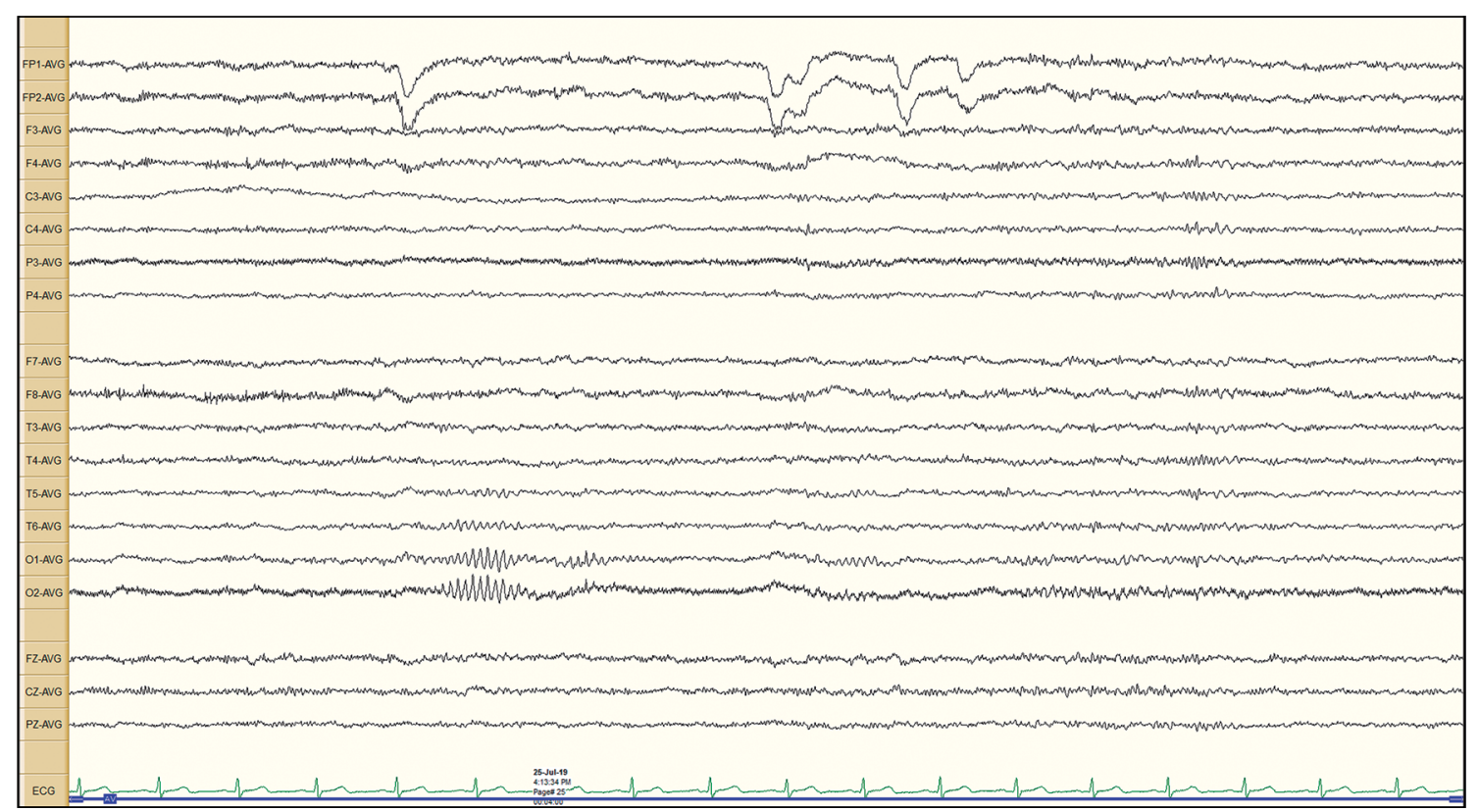

Figura 2. Electroencefalograma (EEG) estándar sin evidencia de alteraciones.

sión progresiva de la alteración de lenguaje y de la paresia. El paciente se hospitalizó en el servicio de medicina interna y luego de $48 \mathrm{~h}$, se logró la disminución de la glicemia a $356 \mathrm{mg} / \mathrm{dl}$, osmolaridad plasmática a $327 \mathrm{mOsm} / \mathrm{kg}$, creatininemia a $1,73 \mathrm{mg} / \mathrm{dL}, \mathrm{pH} 7,35$, bicarbonato de $26,3 \mathrm{mmol} / \mathrm{l}$, kalemia $3,6 \mathrm{mEq} / 1$, natremia $145 \mathrm{mEq} / \mathrm{l}$ y ausencia de cetonas plasmáticas, asociado a remisión total de los síntomas neurológicos focales. Se realizó control con TAC de cerebro a las $48 \mathrm{~h}$ que no evidenció lesiones agudas. El paciente evolucionó sin nueva focalidad y sin secuelas neurológicas. Se complementó estudio metabólico con hemoglobina glicosilada cuyo resultado fue $13,3 \%$, siendo su control de glicemia dificultoso, por lo que fue dado de alta finalmente al décimo día, logrando valores normales de glicemia y osmolaridad. Se indicó esquema de insulina NPH 30 unidades matinales y 16 unidades vespertinas, asociado a metformina $850 \mathrm{mg}$ cada $12 \mathrm{~h}$. En forma ambulatoria, 3 meses después, se realizó un electroencefalograma (EEG) estándar, el cual resultó normal (Figura 2) y se constató adherencia a controles en atención primaria de salud con una hemoglobina glicosilada de $9,2 \%$. Se realizó consentimiento informado para la presentación de este caso.

\section{Discusión}

Las manifestaciones neurológicas del $\mathrm{EHH}$ que más se han descrito son el compromiso de conciencia, que puede abarcar desde la inatención y desorientación hasta el coma, así como convulsiones (incluyendo estatus epiléptico) y los movimientos anormales, sobre todo la hemicorea-hemibalismo ${ }^{4}$. El desarrollo de focalidad neurológica ictal por hiperglicemia ha sido menos descrita, siendo más frecuente en cuadros de hipoglicemia ${ }^{5,6}$.

La relación entre focalidad neurológica y el EHH no ha sido establecida con claridad. En el $\mathrm{EHH}$, la hiperglicemia y la deshidratación pueden llevar a una hipoperfusión global con alteración de la autorregulación cerebral (sobre todo a nivel estriatal y cortical). Además, puede agravar el daño isquémico y la ruptura de la barrera hematoencefálica, produciendo migración de agua con el consiguiente daño celular ${ }^{2}$. Una hipótesis es la aparición de afasia por actividad comicial. La presencia de convulsiones ha sido reportada en $25 \%$ de los casos de $\mathrm{EHH}$, siendo estas focales en un alto porcentaje (a diferencia de la mayoría de los trastornos metabólicos que se presentan con 
convulsiones tónico-clónicas generalizadas) $)^{7,8}$. La inhibición del ciclo de Krebs llevaría a un hipermetabolismo del ácido gamma-aminobutírico (GABA), lo cual disminuiría el umbral convulsivo que, asociado a la deshidratación, hiperosmolaridad e hipernatremia, actuarían como condiciones transitorias que favorecerían la aparición de crisis. Además, lesiones isquémicas antiguas podrían adquirir características epileptogénicas en contexto de desequilibrio metabólico² ${ }^{2}$.

En este caso, el paciente no tenía antecedentes que hiciesen sospechar alguna lesión estructural secuelar ni era previamente epiléptico. Además, hasta ese momento no se había realizado el diagnostico de diabetes mellitus. El patrón de inicio del síndrome afásico y piramidal orientaron hacia una etiología cerebrovascular, por lo cual se activó el protocolo de revascularización y se realizaron las neuroimágenes correspondientes, que incluyen en nuestro centro, TAC de cerebro sin contraste, angioTAC de cuello y cerebro, y en algunos casos particulares, resonancia magnética de cerebro, donde no se encontraron alteraciones en parénquima ni obstrucción de grandes vasos, por lo cual se desestimó la opción de trombolisis endovenosa o trombectomía mecánica. Además, dentro de los exámenes que se solicitan por protocolo, se incluye la glicemia plasmática, cuyo propósito es pesquisar condiciones que simulen patología cerebrovascular, especialmente la hipoglicemia, y por el aumento de riesgo de transformación hemorrágica al realizar trombolisis endovenosa en pacientes con glicemia elevada. En este caso, el valor de glicemia se encontraba elevado y dentro de los rangos que, junto a las otras alteraciones metabólicas descritas, permitieron diagnosticar un EHH e iniciar la corrección hidroelectrolítica.

El tratamiento del EHH consiste en la hidratación y corrección de balance hidroelectrolítico, además de la reducción de la glicemia y osmolari$\mathrm{dad}^{1}$. El uso de antiepilépticos se reserva para casos en los cuales se evidencia actividad convulsiva, sobre todo motora, o cuando hay evidencia de actividad epiléptica crítica en el registro electroencefalográfico asociado a la clínica ictal ${ }^{7}$. En nuestro caso, el manejo con hidratación y administración en infusión continua de insulina revirtió totalmente los síntomas en $48 \mathrm{~h}$, sin necesidad de uso de antiepilépticos.En conclusión, nos encontramos con un paciente que se presenta con un cuadro de focalidad neurológica ictal como debut de diabetes mellitus tipo 2 con un $\mathrm{EHH}$, con neuroimágenes que no evidencian patología cerebrovascular aguda y recuperación total de los déficits iniciales tras la corrección metabólica e hidroelectrolítica. Esto remarca la inclusión de trastornos de glicemia, especialmente de la hiperglicemia, dentro de los stroke mimics.

\section{Referencias}

1. Karslioglu E, Donihi A, Korytkowski M. Diabetic ketoacidosis and hyperosmolar hyperglycemic syndrome: review of acute decompensated diabetes in adult patients. BMJ. 2019; 365: 11114.

2. Misra UK, J. Kalita J, Bhoi SK, Dubey D. Spectrum of hyperosmolar hyperglycaemic state in neurology practice. Indian J Med Res 2017; 146: S1-S7.

3. Liberman A, Prabhakaran S. Stroke Chameleons and Stroke Mimics in the Emergency Department. Neurology of systemic diseases. Curr Neurol Neurosci Rep 2017; 17: 15.

4. Ji Hyun Lee, Ye An Kim, Joon Ho Moon, Se Hee Min, Young Shin Song, and Sung Hee Choi. Expressive aphasia as the manifestation of hyperglycemic crisis in type 2 diabetes. Korean J Intern Med 2016; 31: 1187-90.

5. Long B, Koyfman A. Clinical mimics: an emergency medicine-focused review of stroke mimics. J Emerg Med 2017; 52 (2): 176-83.

6. Nguye P, Chang J. Stroke mimics and acute stroke evaluation: clinical differentiation and complications after intravenous tissue plasminogen activator. J Emerg Med 2015; 49 (2): 244-52.

7. Sabry azar M, Facanha Silva D, Ballailai Ferraz H, Franco de Andrade L. Complex partial seizures and aphasia as initial manifestations of non-ketotic hyperglycemia. Arq Neuropsiquiatr 1998; 56 (2): 296-9.

8. Manford M, Fuller GN, Wade JP. Silent diabetes: non-ketotic hyperglycaemia presenting as aphasic status epilepticus. J Neurol Neurosurg Psychiatry 1995; 59 (1): 99-100. 